\title{
Projected Rotational Velocities in Galactic OB Stars of Different OB Associations
}

\author{
L.J. Corral ${ }^{1}$, A. Herrero ${ }^{1,2}$, and M.R. Villamariz ${ }^{1}$ \\ ${ }^{1}$ Instituto de Astrofísica de Canarias, Vía Lactea s/n,La Laguna, \\ E-38200, Spain \\ 2 Departamento de Astrofísica, Universidad de la Laguna, Avda. \\ Astrofísico Francisco Sánchez s/n, La Laguna, E-38071, Spain
}

\begin{abstract}
As a first step to study the influence that rotation has in the evolution of the most massive stars, we obtained the projected rotational velocity $(v \sin i)$ of a sample of OB stars from clusters and associations. We found that most of our stars present $v \sin i$ lower than $200 \mathrm{~km} / \mathrm{s}$.
\end{abstract}

\section{Introduction}

In order to evaluate the influence that rotation has in the evolution of the most massive stars, we started a program to observe and analyze the spectra of $\mathrm{OB}$ stars that belong to different $\mathrm{OB}$ associations and clusters. We try to obtain a sample of OB stars with different ages and rotational velocities, in order to get information to be able to disentangle both effects in the evolution of the most massive stars. We observed OB stars in different seasons and with different instrumentation from the clusters NGC 1893, IC 1805, NGC 2244, NGC 6611 and use the observations of stars in Cyg OB 2 presented by Herrero et al. (1999). We obtain $v \sin i$ by fitting a rotational profile to the metallic lines present in the optical spectra of 50 stars. The distribution of the projected rotational velocity vs. spectral type found in each association is presented in Fig. 1a. In Figs. 1b, $1 c$ and $1 \mathrm{~d}$ we present the same data but for each luminosity class. We compare these velocities with the mean rotational velocities of different groups of stars presented by Fukuda (1982).

The first thing that we notice is that high velocity $(v \sin i>200 \mathrm{~km} / \mathrm{s})$ main sequence objects are missing. The most rapid rotator among our early $O$ type main sequence objects presents a $v \sin i=200 \mathrm{~km} / \mathrm{s}$, just a little higher that the mean velocity of objects earlier than $\mathrm{O} 7 \mathrm{~V}(178 \mathrm{~km} / \mathrm{s})$ in Fukuda's sample. All late $\mathrm{O}$ type stars observed present $v \sin i$ smaller than the mean $(158 \mathrm{~km} / \mathrm{s})$, and almost all B V objects have $v \sin i$ smaller than the mean $(\sim 150 \mathrm{~km} / \mathrm{s})$

In the case of objects with luminosity class III, 3 of our 4 objects earlier than O7 have $v \sin i$ larger than the corresponding mean quoted by Fukuda (1982) that is of $91 \mathrm{~km} / \mathrm{s}$, but this mean rotational velocity was obtained from a single object and it can not be taken as representative of the group. All of our late type $\mathrm{O}$ giants have projected rotational velocities smaller than the mean (150 $\mathrm{km} / \mathrm{s}$ ) and this is also the case for the early B giants, except for one of them that has $v \sin i$ slightly larger than the mean $(108 \mathrm{~km} / \mathrm{s})$. 

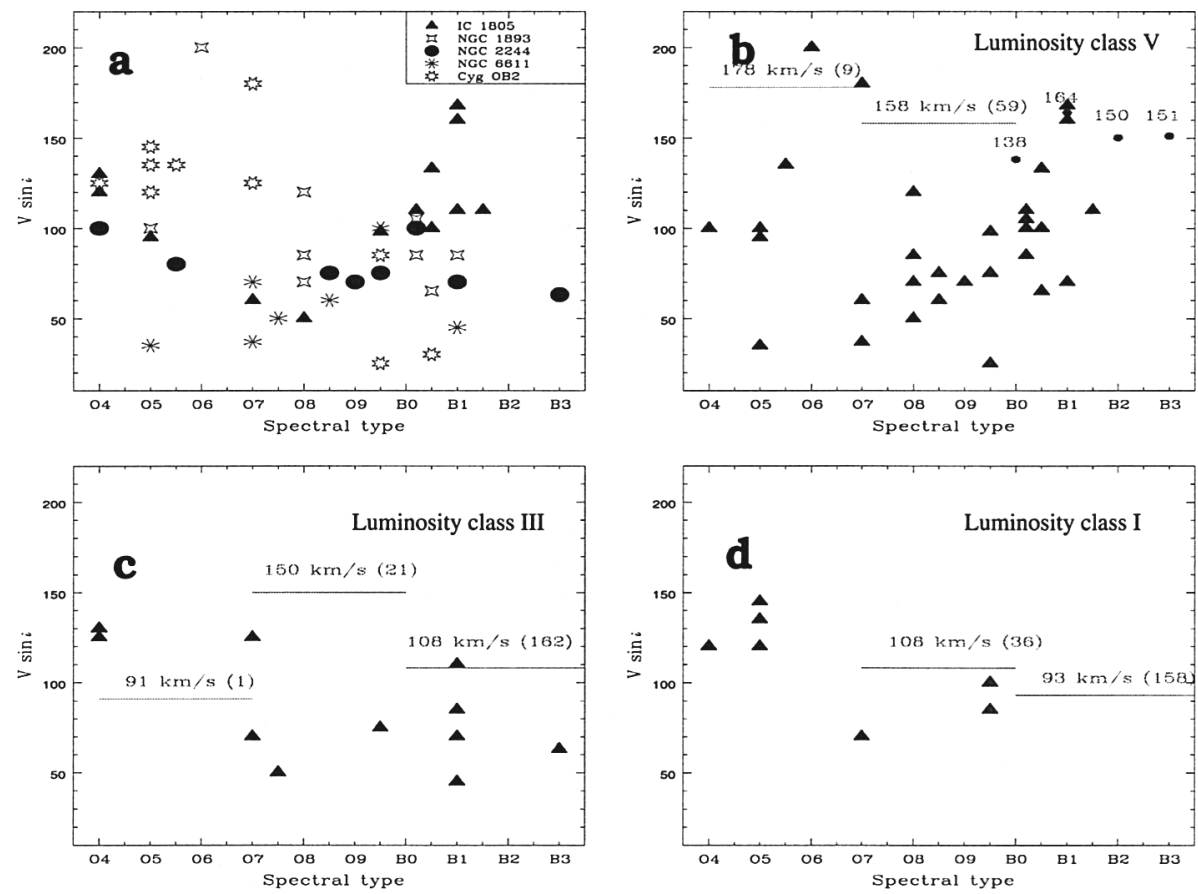

For the objects classified as early $\mathrm{O}$ type supergiants (Luminosity class I) Fukuda (1982) does not present the mean projected rotational velocity, but using data from Penny (1996) we found an average $v \sin i$ of $148 \mathrm{~km} / \mathrm{s}$ for objects earlier than $\mathrm{O} 7$, while the stars that we observed present smaller rotational velocities. We obtain the same results with those objects classified as late $\mathrm{O}$ type supegiants where the mean projected rotational velocity of Fukuda (1982) is $108 \mathrm{~km} / \mathrm{s}$.

These results can be produced by the lack of observations of all objects in the sample or if stars formed in clusters have a preferential orientation of the $v_{\text {rot }}$ vector. A priori we do not expect this latter case if we assume that turbulent motions in the parent clouds that formed these stars prevailed over motions produced by global magnetic fields or galactic differential rotation, that can give a preferential direction of angular momentum.

\section{References}

Fukuda, I. 1982 PASP 94, 27

Herrero, A., Corral, L.J., Villamariz, R., Martn, E.L. 1999 A\&A 348, 542.

Hillenbrand, L, Massey, P., Strom, S., Merrill, M. 1993 AJ 106, 1906.

Massey, P., Johnson, K., Degioia-Eastwood, K. 1995 ApJ 454, 151. 